\title{
Objective Assessment of Left Ventricular Wall Motion from Cardiac Magnetic Resonance Images
}

\author{
EG Caiani ${ }^{1,2}$, E Toledo ${ }^{1}$, P MacEneaney ${ }^{1}$, KA Collins ${ }^{1}$, RM Lang ${ }^{1}$, V Mor-Avi ${ }^{1}$ \\ ${ }^{1}$ Department of Medicine, University of Chicago, Chicago, IL, USA \\ ${ }^{2}$ Dipartimento di Bioingegneria, Politecnico di Milano, Milan, Italy
}

\begin{abstract}
Cardiac MRI diagnosis of regional LV dysfunction relies on subjective interpretation of cine images that suffers from wide inter-observer variability, especially when performed by readers not trained in the assessment of regional wall motion. We developed a method for objective, automated detection of regional wall motion abnormalities. Cine images (GE 1.5T, FIESTA) were obtained in 6-10 short axis slices in 10 pts. Images were reviewed by an expert cardiologist whose grades were used as the gold standard. Images were then analyzed using custom software. In each slice, endocardial border was detected semi-automatically and divided into 6 segments. Regional fractional area change (RFAC) was computed and displayed as "bull's eyes". RFAC $<50 \%$ was considered abnormal. The "bull's eyes" showed dark areas in segments judged as abnormal by the expert reader. The automated detection had sensitivity 79\%, specificity $78 \%$, accuracy $78 \%$. Analysis of endocardial motion from MRI images provides the basis for accurate, automated and objective interpretation of regional wall motion.
\end{abstract}

\section{Introduction}

Cardiac magnetic resonance (CMR) based diagnosis of regional left ventricular (LV) dysfunction relies on subjective and experience dependent visual interpretation of dynamic images in multiple planes. This interpretation is often performed by radiologists who do not have the specialized training and experience necessary for accurate diagnosis of regional wall motion abnormalities. Thus, the outcome of this methodology may vary widely between observers [1]. Our goal was to test the feasibility of objective detection of regional wall motion abnormalities based on quantitative segmental analysis of semi-automatically defined LV endocardial boundaries. The performance of this technique was tested by comparing the results of automated detection with the results of conventional visual interpretation of $\mathrm{LV}$ wall motion by an expert reader. To put these results in perspective, we also studied the accuracy and the interobserver variability of the conventional visual interpretation by less experienced readers on the same set of images.

\section{Methods}

Ten consecutive patients (age 52 \pm 14 years, 6 men, 4 women) referred for CMR evaluation of LV function were studied. Exclusion criteria were: cardiac arrhythmias, left bundle branch block, prior sternotomy, pacemaker or defibrillator implantation, claustrophobia and other known contraindications to MR imaging.

\subsection{CMR image acquisition}

CMR images were obtained using a 1.5 Tesla scanner (General Electric) with a phased-array cardiac coil. ECGgated, steady-state free-precession dynamic gradient-echo (FIESTA) cine-loops were obtained during $12 \mathrm{sec}$ breathholds at 20 frames per cardiac cycle. In all patients, 6 to 10 short-axis cine-loops were obtained from base to apex (9 mm slices, no gaps).

\subsection{Visual interpretation of wall motion}

Dynamic images were reviewed by an expert cardiologist, who graded regional LV wall motion in each segment (6 segment model) in each slice as normal or abnormal. These expert grades were used as the "gold standard" for comparisons.

In addition, the same images were independently reviewed and graded by two general radiologists with minimal experience in the interpretation of regional $\mathrm{LV}$ function. The sensitivity, specificity and accuracy of each of the two readers were calculated against the "gold standard" grades on a segment-by-segment basis. In addition, the inter-observer variability was calculated from the radiologists' interpretations as percent of segments with discordant grades. 

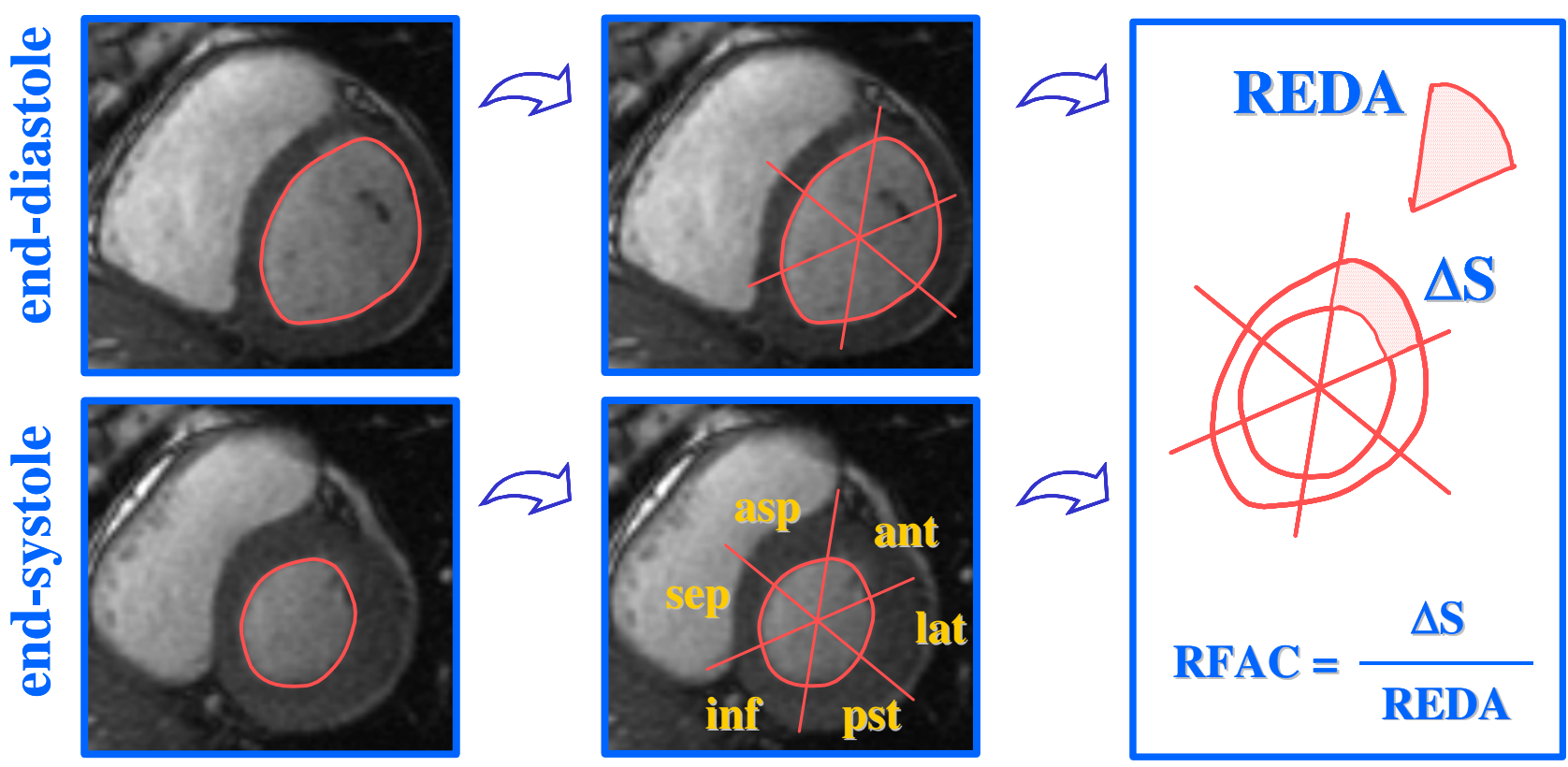

Figure 1. Endocardial border detection on end-diastolic (ED, top, left) and end-systolic (ES, bottom, left) frames followed by segmentation and calculation of regional fractional area change (RFAC), which was defined for each segment as the area $\triangle \mathrm{S}$ between the ED and ES position of the endocardial border, divided by the regional end-diastolic area (REDA).

\subsection{Computer analysis of CMR images}

In each slice, endocardial border was semiautomatically detected frame-by-frame throughout the cardiac cycle. This was achieved by adaptive thresholding of videointensity based on pixel position. A binary image was created with a pixel position-dependent threshold [2], and further processed with standard morphological operators to extract the LV cavity. Thresholding parameters were initially adjusted for the first frame in each image sequence by visually verifying the position of the resulting contour, which was superimposed on the original image. Then, the optimal parameters were applied to the subsequent images, and adjusted when necessary to optimize border position for that particular frame.

LV cavity area was then calculated frame-by-frame directly from pixel counts inside the detected endocardial border. End-systolic and end-diastolic frames were automatically identified as those with the smallest and largest LV area (figure 1, left). The LV cavity was then divided into six $60^{\circ}$ wedge-shaped segments (figure 1, middle), using a standard segmentation scheme for the LV short-axis view [3], corresponding to myocardial segments used for visual assessment and grading of wall motion. For each segment, regional fractional area change (RFAC) in \% of regional end diastolic area (REDA) was calculated automatically (figure 1, right) [4].

\subsection{Automated interpretation of wall motion}

In each segment, the calculated RFAC value was used as a quantitative index of regional systolic endocardial motion for automated detection of wall motion abnormalities (figure 2). Segments where RFAC was above $50 \%$ were automatically classified as normal (n), and those below 50\% were classified as abnormal (a). The concordance rates with the "gold standard" expert grades were used to calculate the sensitivity, specificity and overall accuracy of the automated interpretation of regional wall motion.

In addition, RFAC values were displayed in a "bull's eye" format, similar to that used in nuclear cardiology, to allow easy visualization of the wall motion patterns in the entire ventricle in a single picture. In this display, the inner circle represent the apex, while the rings represent consecutive CMR slices from apex to base.
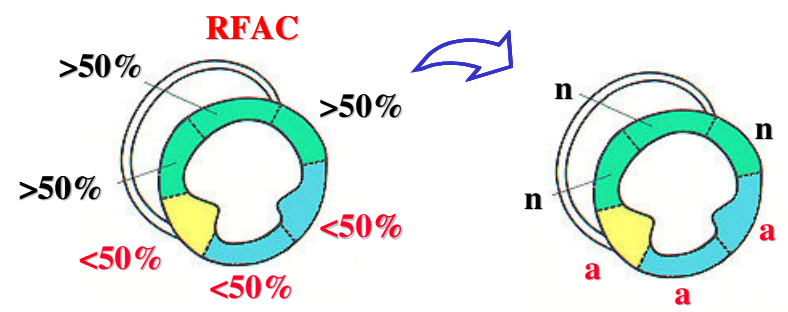

Figure 2. Automated detection of wall motion abnormalities (see text for details). Colors represent different vascular territories. 

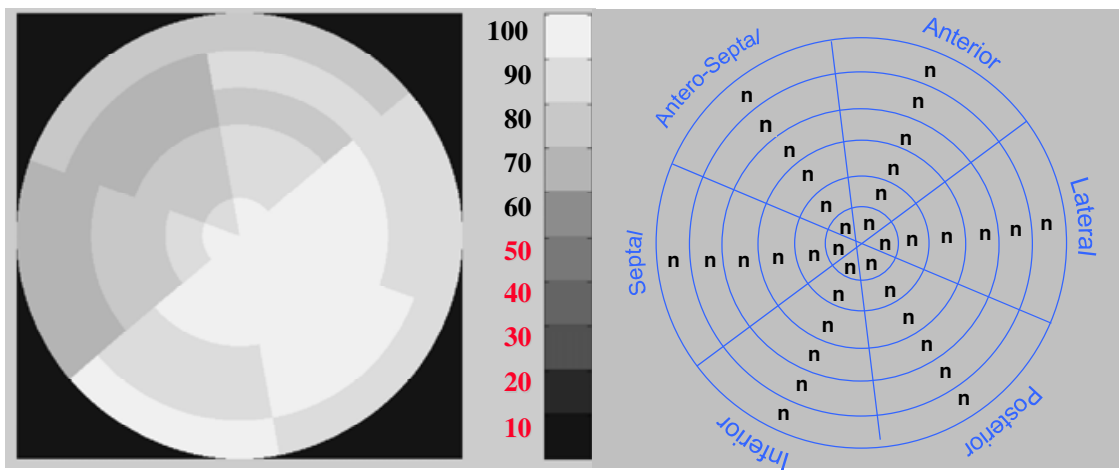

Figure 3. Example of data obtained in a patient with normal LV wall motion: a "bull's eye" display of RFAC (left) and a summary of the "gold standard" grades in the corresponding segments (right).

\section{Results}

The expert reader detected abnormal wall motion in 7 out of 10 patients. Of the total 434 segments studied in the 10 patients, wall motion in 140 segments (32\%) was graded as abnormal.

The calculation of RFAC was fully automated and required $<1 \mathrm{sec}$ per slice on a $2 \mathrm{GHz}$ Pentium 4 personal computer. In patients with normal wall motion according to the "gold standard" interpretation, the "bull's eye" display of RFAC showed relatively uniform colordistribution, reflecting RFAC $>50 \%$ in the entire ventricle (figure 3). In patients with wall motion abnormalities, the "bull's eye" showed dark areas reflecting reduced RFAC $(<50 \%)$ in segments corresponding to abnormalities detected by the expert reviewer (figure 4 ).

The automated technique showed abnormally reduced RFAC in 169/434 segments (39\%) and normal RFAC in the remaining 265/434 segments (61\%). This interpretation disagreed with the "gold standard" in $92 / 434$ segments $(21 \%)$, resulting in overall accuracy of $79 \%$. Of these 92 discordant segments, 64 were false positive, and 28 were false negative, resulting in a sensitivity of $79 \%$ and specificity of $78 \%$.

The radiologists' grades were discordant between them in 114/434 segments $(26 \%)$, reflecting the high interobserver variability of the non-expert readers. Compared to the "gold standard", their grades resulted in an average accuracy of $73 \%$ (table 1 ).
Table 1. Performance of visual interpretation of LV wall motion by two general radiologists, compared to a "gold standard" read provided by an expert cardiologist.

\begin{tabular}{|l|c|c|c|}
\hline & Sensitivity & Specificity & Accuracy \\
\hline Observer 1 & $93 \%$ & $52 \%$ & $65 \%$ \\
\hline Observer 2 & $91 \%$ & $75 \%$ & $80 \%$ \\
\hline Average & $92 \%$ & $64 \%$ & $73 \%$ \\
\hline
\end{tabular}

\section{Discussion and conclusions}

Clinical assessment of LV wall motion is mostly based on visual interpretation of dynamic ultrasound and, more recently, CMR images. This methodology heavily relies on the reader's ability to efficiently extract and integrate spatial and temporal information on endocardial motion and wall thickening, which requires extensive training and is known to be subjective and experience dependent $[5,6]$. While echocardiographic images are usually acquired in dedicated cardiac imaging laboratories and interpreted by cardiologists trained in the evaluation of LV wall motion, CMR imaging is commonly performed in radiology departments and images are reviewed by physicians without dedicated training and extensive experience with this methodology. As a result, the
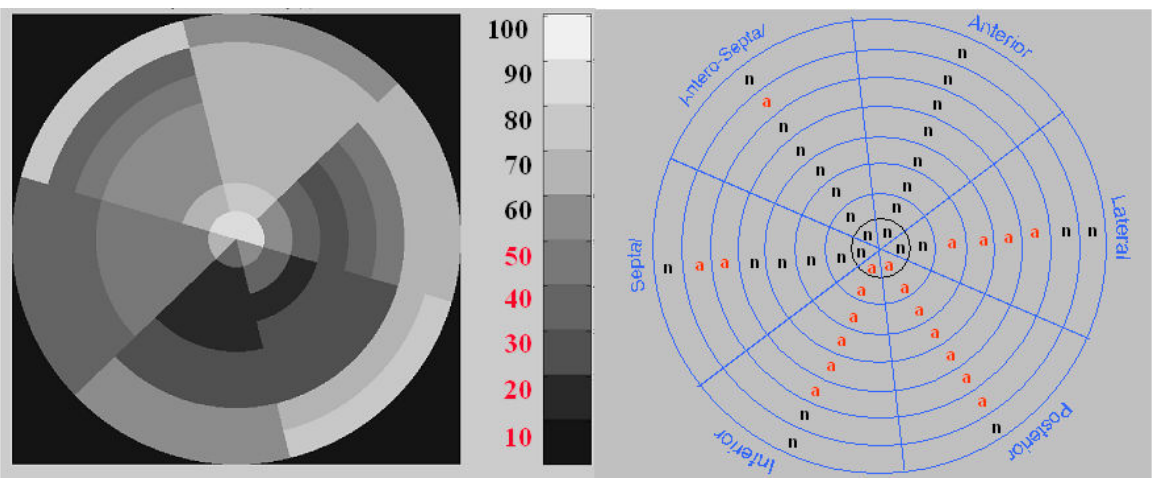

Figure 4. Example of data obtained in a patient with abnormal LV wall motion. Data presented in the same format as in figure 3 . 
detection of regional wall motion abnormalities from CMR images may be less accurate and may suffer from high inter-observer variability, as indeed confirmed by our results.

In the present study, we developed and tested a new technique for automated interpretation of LV wall motion based on the calculation of RFAC. Although this technique uses semi-automatically traced endocardial contours, which is likely to introduce a certain level of inter-observer variability, its major strength is that the interpretation is fully automated and therefore objective and experience-independent. In addition, the goal of this study was to test the initial feasibility of automated interpretation of regional wall motion from CMR images and its accuracy against expert interpretation, rather than calculate the inter-observer variability introduced by semi-automatic endocardial tracing.

Our results showed reasonably high levels of sensitivity, specificity and accuracy, comparable to those of other clinically used techniques. These results were achieved despite the fact that we used an arbitrarily set $50 \%$ RFAC threshold for detection of wall motion abnormalities. It is likely that higher levels of accuracy could be reached by optimizing threshold selection for each individual slice and segment, since regional motion patterns vary from segment to segments and from slice to slice. This will need to be achieved by acquiring and analyzing data in a large group of normal subjects, necessary to establish a normal range of RFAC for each segment [7].

Importantly, the automated technique provided more accurate interpretation of regional ventricular wall motion than the radiologists' reads. This improved accuracy was accompanied by another important advantage, i.e. zero inter-observer variability. In addition, our technique resulted in similar sensitivity and specificity levels, as opposed to the radiologists' interpretation that had high sensitivity on the expense of low specificity.

In conclusion, quantitative analysis of regional endocardial motion from CMR images is feasible and provides the basis for accurate detection of regional wall motion abnormalities. Since semi-automated border detection is available in most commercial analysis software packages, this approach can be easily implemented into the existing tools and promises to become a clinically valuable addition in the diagnosis of ischemic heart disease.

\section{References}

[1] Caiani EG, Toledo E, MacEneaney P, Collins KA, Lang RM, Mor-Avi V: The role of still-frame parametric imaging in magnetic resonance assessment of left ventricular wall motion by non-cardiologists. J Cardiovasc Magnetic Resonance 2004; 6:619-25

[2] Mor-Avi V, Caiani EG, Collins KA, Korcarz C, Bednarz JE, Lang RM: Combined assessment of myocardial perfusion and regional left ventricular function using quantitative analysis of contrast-enhanced power modulation images. Circulation 2001; 104:352-357

[3] Schiller NB, Shah PM, Crawford M, DeMaria A, Devereux $\mathrm{R}$, Feigenbaum $\mathrm{H}$ et al. Recommendations for quantitation of the left ventricle by two-dimensional echocardiography. J Am Soc Echocardiogr 1989; 2:358-367

[4] Mor-Avi V, Vignon P, Koch R, Weinert L, Garcia MJ, Spencer K, Lang RM: Segmental analysis of Color Kinesis images: New method for quantitative assessment of left ventricular contraction and relaxation. Circulation 1997; 95:2082-2097

[5] Popp RL, Agatston A, Armstrong WF, Nanda NC, Pearlman A, Rakowski et al. Recommendations for training in performance and interpretation of stress echocardiography. $\mathbf{J}$ Am Soc Echocardiogr 1998; 11:95-96

[6] Picano E, Lattanzi F, Orlandini A, Marini C, L'Abbate A. Stress echocardiography and the human factor: the importance of being expert. J Am Coll Cardiol 1991; 17:666-669

[7] Mor-Avi V, Spencer K, Gorcsan J, DeMaria A, Kimball T, Monaghan M, Perez J, Sun JP, Weinert L, Bednarz J, Collins K, Edelman K, Kwan OL, Glascock B, Hancock J, Baumann C, Thomas J, Lang R: Normal values of regional left ventricular endocardial motion: multicenter color kinesis study. Am J Physiol 2000; 279:H2464-H2476

Address for correspondence:

Victor Mor-Avi, Ph.D.

University of Chicago Medical Center, MC5084, 5841 S.

Maryland Ave., Chicago Illinois 60637, USA

Email: vmoravi@medicine.bsd.uchicago.edu 